\section{Fetal brain lesions after sub- cutaneous inoculation of Zika virus in a pregnant nonhuman primate}

Kristina M Adams Waldorf ${ }^{1}$, Jennifer E Stencel-Baerenwald ${ }^{2,3,21}$, Raj P Kapur ${ }^{4,5,21}$, Colin Studholme ${ }^{6-8,21}$, Erica Boldenow $6,9,20,21$, Jay Vornhagen $^{9,10,21}$, Audrey Baldessari ${ }^{11}$, Manjiri K Dighe ${ }^{8}$, Jeff Thiel $^{8}$, Sean Merillat ${ }^{9}$, Blair Armistead ${ }^{9,10}$, Jennifer Tisoncik-Go ${ }^{2,3}$, Richard R Green ${ }^{2,3}$, Michael A Davis ${ }^{2,3}$, Elyse C Dewey ${ }^{2,3}$, Marian R Fairgrieve ${ }^{2,3}$, J Christopher Gatenby ${ }^{8}$, Todd Richards ${ }^{8}$, Gwenn A Garden ${ }^{4,12}$, Michael S Diamond ${ }^{13-16}$, Sandra E Juul ${ }^{6}$, Richard F Grant ${ }^{11}$, LaRene Kuller ${ }^{11}$, Dennis W W Shaw ${ }^{8,17}$, Jason Ogle ${ }^{11}$, G Michael Gough ${ }^{11}$, Wonsok Lee ${ }^{11}$, Chris English ${ }^{11}$, Robert F Hevner ${ }^{18,19}$, William B Dobyns ${ }^{6,19}$, Michael Gale Jr ${ }^{2,3}$ \& Lakshmi Rajagopal $6,9,10$

We describe the development of fetal brain lesions after Zika virus (ZIKV) inoculation in a pregnant pigtail macaque. Periventricular lesions developed within $10 \mathrm{~d}$ and evolved asymmetrically in the occipital-parietal lobes. Fetal autopsy revealed ZIKV in the brain and significant cerebral white matter hypoplasia, periventricular white matter gliosis, and axonal and ependymal injury. Our observation of ZIKV-associated fetal brain lesions in a nonhuman primate provides a model for therapeutic evaluation.

The recent ZIKV epidemic in the Americas has been declared a global public health emergency because of its association with severe fetal brain injury during infection of pregnant women. ZIKV is a mosquitotransmitted flavivirus that generally causes a self-limited febrile syndrome characterized by rash, conjunctivitis and arthralgia. However, infection in pregnant women is a major concern because the fetus may develop a range of brain anomalies, including cerebral atrophy, ventriculomegaly, cerebellar hypoplasia and ocular manifestations ${ }^{1}$. Although ZIKV infection can induce fetal demise in mice ${ }^{2-4}$, the lack of an experimental animal model of ZIKV infection that closely emulates human pregnancy has hindered the ability to directly test the causal relationship between ZIKV infection and fetal brain injury ${ }^{5}$. Here we describe the development of a pregnant pigtail macaque model of ZIKV infection that results in fetal brain lesions. Our results confirm the teratogenic potential of ZIKV and will enable testing of therapeutics to prevent fetal brain injury.

We inoculated ZIKV (strain FSS13025, Cambodia 2010) subcutaneously in five locations on the forearms of a pregnant pigtail macaque at $119 \mathrm{~d}$ gestation (corresponding to $\sim 28$ weeks of human pregnancy) to test whether ZIKV causes fetal brain injury. Each of the five inoculations consisted of $10^{7}$ plaque-forming units (p.f.u.) of ZIKV. The pregnant animal appeared healthy and did not develop signs of rash, conjunctivitis or fever. Cesarean section was performed before the onset of labor at $162 \mathrm{~d}$ gestation (corresponding to $\sim 38$ weeks of human pregnancy). A weekly ultrasound demonstrated a lag in the growth of the fetal biparietal diameter (BPD) (Fig. 1a) that ultimately fell three standard deviations as compared to the published ultrasound data for fetal pigtail macaques ${ }^{6}$. In contrast, the femur continued to grow normally (Fig. 1b). Baseline imaging of the fetal head showed normal architecture of the cerebral hemispheres, ventricles and posterior fossa; over time, a heterogeneous linear echogenic area developed adjacent to the lateral ventricle in the posterior brain of the ZIKV-infected animal (Fig. 1c) that was not seen in a control fetus at a similar stage of gestation (Fig. 1d).

The first magnetic resonance imaging (MR imaging, or MRI) of the fetal brain was performed $10 \mathrm{~d}$ following ZIKV inoculation and demonstrated bilateral periventricular T2 hyperintense foci in the occipitalparietal regions surrounding the occipital horns of the lateral ventricles (Fig. 1e and Supplementary Figs. 1-3). This abnormality evolved over the course of the study and enlarged on the right; on the left, the T2 hyperintense focus became hypointense and was associated with volume loss and ventricular collapse. White matter volume did not change significantly over the last 3 weeks of the study (17-43 d after inoculation), which correlated with the plateau in growth of the biparietal diameter on the ultrasound image; in contrast, the gray matter consistently increased in volume (Fig. 1a, Supplementary Fig. 4 and Supplementary Table 1). The white matter surface area, shape and cortical folding continued to evolve (Supplementary Figs. 4 and 5 and Supplementary Table 1). Normally, during the latter half of pregnancy in the macaque, the posterior ventricular cerebrospinal fluid regions reduce in size relative to the more rapid development

\footnotetext{
${ }^{1}$ Department of Obstetrics and Gynecology, University of Washington, Seattle, Washington, USA. 2Department of Immunology, University of Washington, Seattle, Washington, USA. ${ }^{3}$ Center for Innate Immunity and Immune Disease, University of Washington, Seattle, Washington, USA. ${ }^{4}$ Department of Pathology, University of Washington, Seattle, Washington, USA. ${ }^{5}$ Department of Pathology, Seattle Children's Hospital, Seattle, Washington, USA. ${ }^{6}$ Department of Pediatrics, University of Washington, Seattle, Washington, USA. ${ }^{7}$ Department of Bioengineering, University of Washington, Seattle, Washington, USA. ${ }^{8}$ Department of Radiology, University of Washington, Seattle, Washington, USA. ${ }^{9}$ Center for Global Infectious Disease Research, Seattle Children's Research Institute, Seattle, Washington, USA. ${ }^{10}$ Department of Global Health, University of Washington, Seattle, Washington, USA. ${ }^{11}$ Washington National Primate Research Center, Seattle, Washington, USA. ${ }^{12}$ Department of Neurology, University of Washington, Seattle, Washington, USA. ${ }^{13}$ Department of Medicine, Washington University School of Medicine, St. Louis, Missouri, USA. ${ }^{14}$ Department of Molecular Microbiology, Washington University School of Medicine, St. Louis, Missouri, USA. ${ }^{15}$ Department of Pathology and Immunology, Washington University School of Medicine, St. Louis, Missouri, USA. ${ }^{16}$ Center for Human Immunology and Immunotherapy Programs, Washington University School of Medicine, St. Louis, Missouri, USA. ${ }^{17}$ Department of Radiology, Seattle Children's Hospital, Seattle, Washington, USA. ${ }^{8}$ Department of Neurological Surgery, University of Washington, Seattle, Washington, USA. ${ }^{19}$ Center for Integrative Brain Research, Seattle Children's Research Institute, Seattle, Washington, USA. ${ }^{20}$ Present address: Department of Biology, Calvin College, Grand Rapids, Michigan, USA. ${ }^{21}$ These authors contributed equally to this work. Correspondence should be addressed to K.M.A.W. (adamsk@uw.edu), M.G. (mgale@uw.edu) or L.R. (lakshmi.rajagopal@seattlechildrens.org).
}

Received 17 June; accepted 31 August; published online 12 September 2016; doi:10.1038/nm.4193 

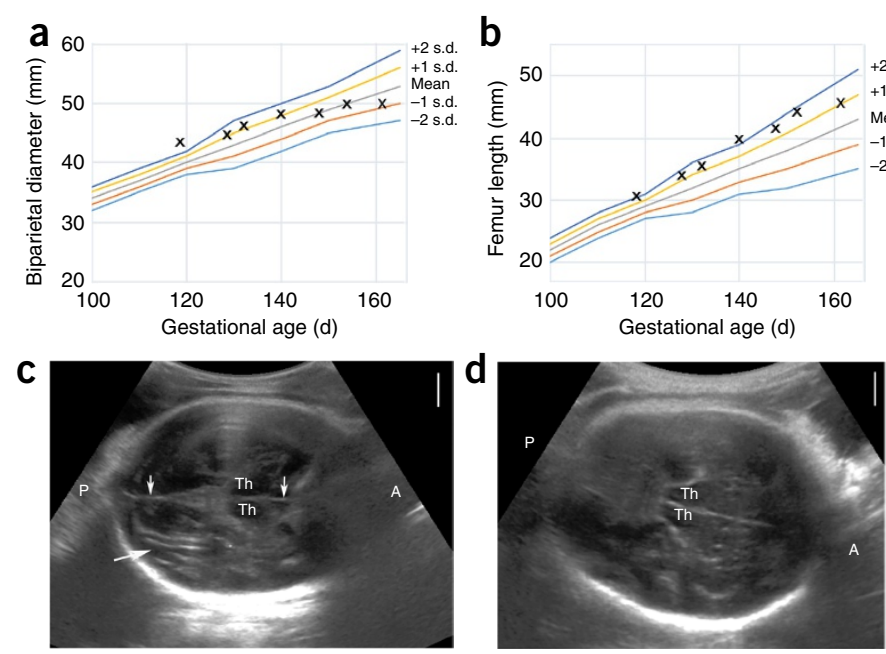

e

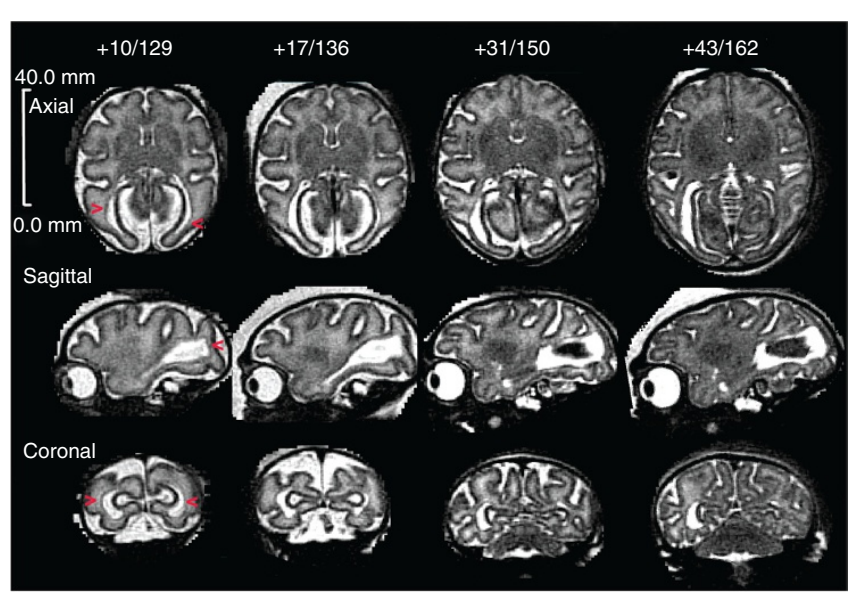

Figure 1 Fetal biometry, ultrasound and serial fetal brain MR imaging of a ZIKV-infected pregnant pigtail macaque. (a,b) Fetal biparietal diameter (BPD) (a) and femur length (b) of a ZIKV-infected pregnant pigtail macaque over time, as measured by ultrasound and compared to published reference data for fetal growth; each individual line refers to the mean and standard deviation (s.d.) extrapolated from the tables in the reference for the pigtail macaque 6 . (c) An axial image of the fetal head obtained by ultrasound at $161 \mathrm{~d}$ gestation from the ZIKV-infected fetus. The large white arrow indicates a heterogeneous linear echogenic area in the posterior cerebral cortex on the right side. Other visible structures include the thalamus (Th) and falx (small white arrows). Labels indicate the anterior (A) and posterior (P) orientation. (d) An analogous image to $\mathbf{c}$ is shown for a control fetus at $163 \mathrm{~d}$ gestation. In c,d, scale bars, $1 \mathrm{~cm}$. (e) Serial axial (top), sagittal (middle) and coronal (bottom) MR images of the fetal brain in a ZIKVinfected pregnant pigtail macaque from 10 to $43 \mathrm{~d}$ after inoculation (129-162 d gestation). The red arrowheads indicate T2 hyperintense foci in the bilateral periventricular regions of the occipital-parietal brain. Numbers shown at the top of the image indicate 'time after inoculation (d)/time of gestation (d)'. Scale bar, $40 \mathrm{~mm}$ (top).

of the parietal and occipital lobes. In contrast, after ZIKV inoculation large posterior ventricular cerebrospinal fluid spaces persisted into late gestation. The surrounding posterior white matter also showed enhancement in T2 weighting, indicating increased water content (Supplementary Table 1). There was no evidence of cortical malformation or abnormalities of the brainstem or cerebellum by MR imaging. MR spectroscopy demonstrated a marked reduction in $\mathrm{N}$-acetyl aspartate (NAA), a metabolite associated with neuronal integrity, in the posterior versus the anterior brain (Supplementary Fig. 6). The rapid development of an enhancing T2-weighted fluid abnormality suggests that fetal brain injury begins to occur quickly following ZIKV infection and may induce periventricular white matter injury similar to that seen after infection with other viruses that cause neonatal encephalitis ${ }^{7,8}$.

Macroscopic examination of the ZIKV-infected fetal brain revealed marked deficiency of the posterior white matter as compared to the control fetal brain (Fig. 2a,b). White matter gliosis was present bilaterally with many apoptotic and mitotic figures in contrast to the control fetus (Fig. 2c-f). Immunohistochemistry revealed increased density of glial fibrillary acidic protein (GFAP)-positive reactive astrocytes, predominantly in the white matter, as compared to the control fetus (Fig. 2g,h), and increased CD163-positive microglial cells (data not shown). Flow cytometry analysis of matched regions of the fetal and maternal brain indicated an enrichment of astrocytes in the fetal brain (Supplementary Table 2). Ovoid eosinophilic structures were seen in the gliotic white matter that were consistent with axonal spheroids (Fig. 2f, inset), a pathologic sign of axon injury. The occipital horn of the right lateral ventricle was mildly dilated. There was mild focal gliosis in the gray matter. Notably, there was bilateral patchy loss of ventricular ependymal epithelium with underlying parenchymal hypercellularity (Supplementary Fig. 7b) and increased activated caspase-3 immunoreactivity (Supplementary Fig. 7d); CD163-positive histiocytes adhered to the denuded ventricle surface (data not shown). In contrast, few caspase-3-immunoreactive cells were present in the periventricular zone of an uninfected fetal control (Supplementary Fig. 7c). Minimal inflammation was observed in the brain parenchyma and meninges; CD20- and CD3-positive cells were rare (not shown). Necrosis, hemorrhage or calcifications were absent. Other maternal and fetal organs, including the fetal brainstem, did not show pathological signs of injury. Placental histopathology revealed only mild deciduitis, which was also present in some uninfected controls.

Prior to inoculation, serology was negative for previous infection with ZIKV, dengue virus (DENV), West Nile virus (WNV) or chikungunya virus (CHIKV) (data not shown). The dam became seropositive for ZIKV-specific IgG at $14 \mathrm{~d}$ after inoculation and remained positive; the fetus was seropositive at birth (Supplementary Table 3). At delivery, ZIKV RNA was detected in the chorionic villous tissue of the placenta, the fetal brain and liver, and the maternal brain, eyes, spleen and liver (Fig. 2i and Supplementary Table 4). Whole-transcriptome sequencing analysis of brain tissues identified ZIKV RNA in both fetus (1,298 reads per $\mathrm{mg}$ tissue) and dam (340 reads per mg tissue), with sequence-read coverage across the complete ZIKV genome, including the nontranslated regions (Fig. 2j). No viral sequences were identified in the brain of an uninfected female pigtail macaque. Viral plaque assays on all tissues with detectable ZIKV RNA were negative for replicating virus, indicating that either the infection had resolved or the level of infectious virus was too low for recovery using standard plaque assay methodology.

Our findings of arrested fetal brain growth and viral neuro-invasion are consistent with the congenital ZIKV syndrome seen in humans. The marked lag in fetal BPD growth by ultrasound analysis reflects the arrest of white matter expansion detected by MR imaging. A similar arrest in BPD has been reported in human pregnancy with presumed ZIKV infection in the third trimester ${ }^{9}$. In the context of ZIKV infection, this change in fetal brain growth would be very concerning for 


\section{BRIEF COMMUNICATIONS}

a

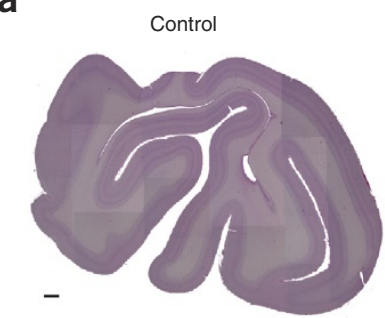

C

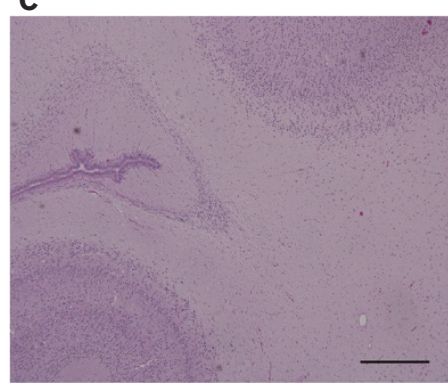

e

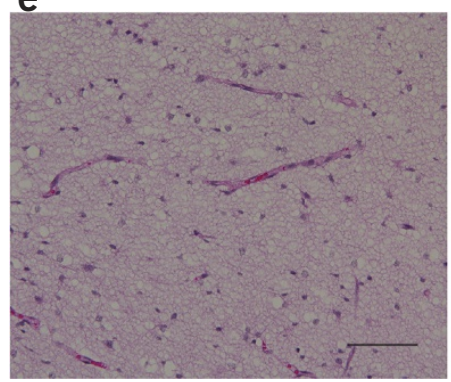

g

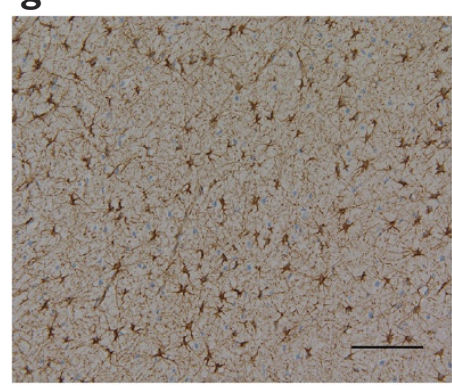

b

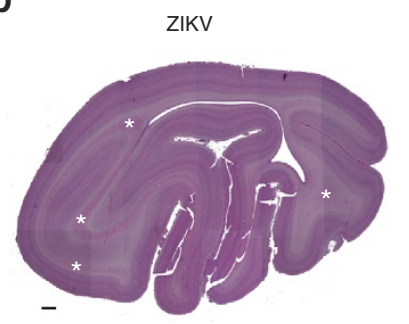

d

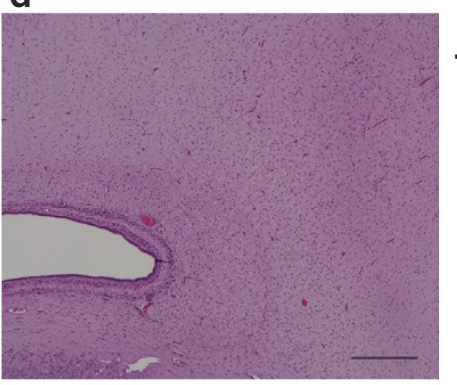

f

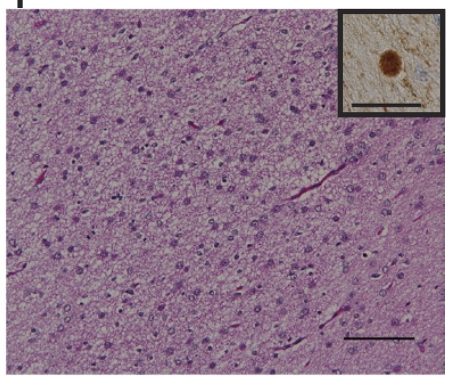

h

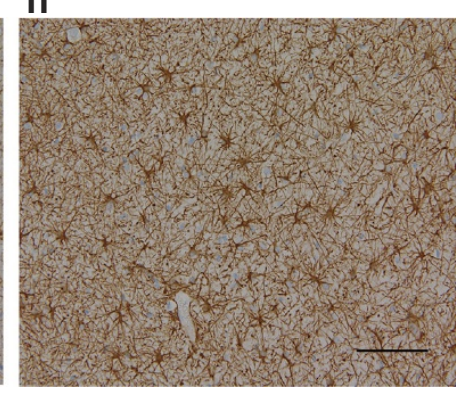

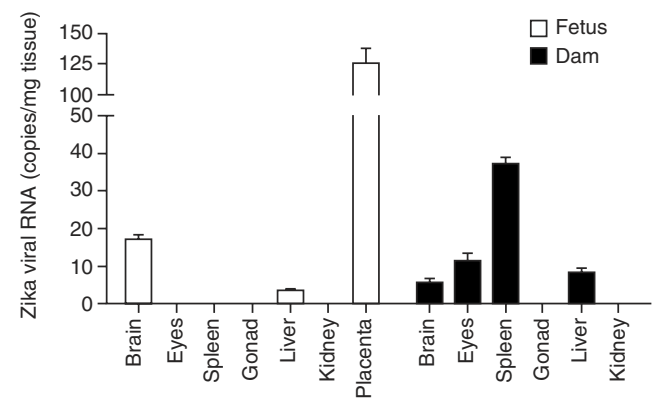

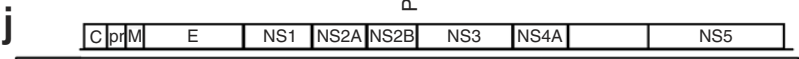
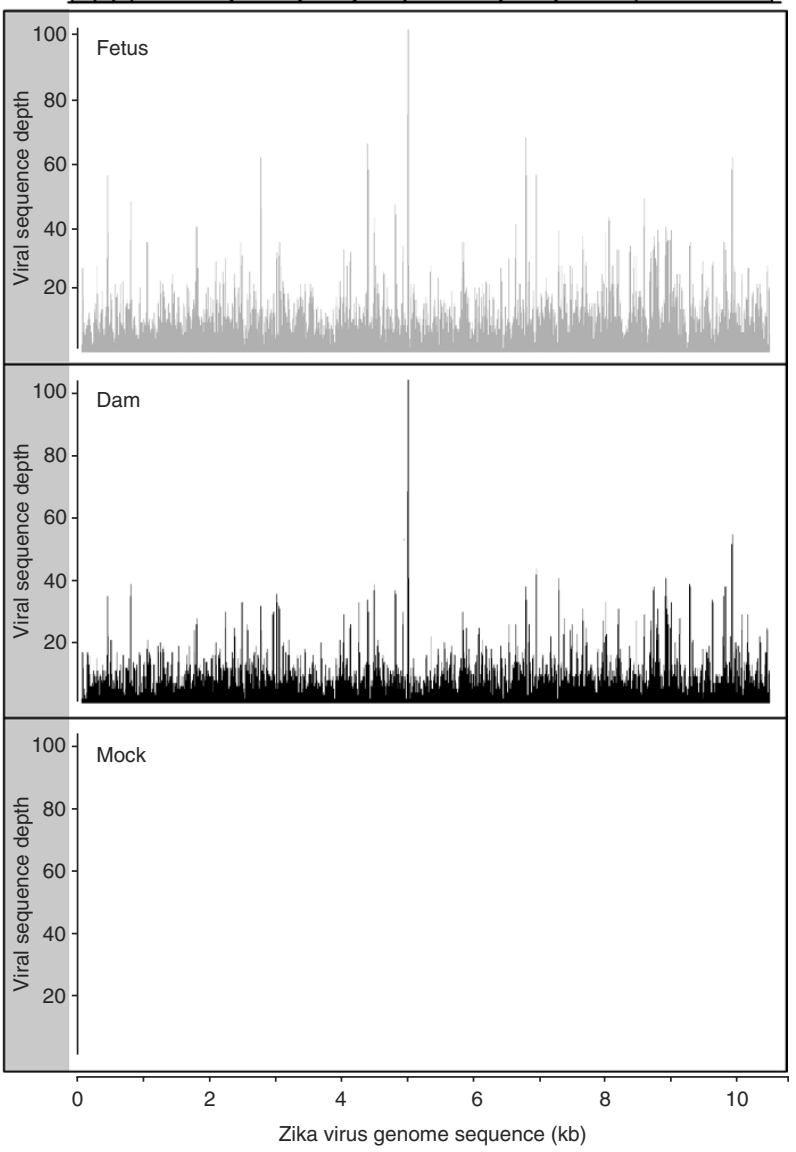

Figure 2 Neuropathology of the ZIKV-infected fetal brain and ZIKV RNA in fetal and dam tissues. (a-f) Representative H\&E-stained images of the brains from one ZIKV-infected fetus (b,d,f) and a control fetus $(\mathbf{a}, \mathbf{c}, \mathbf{e})$ are shown. White asterisks $(\mathbf{b})$ indicate pink bands of white matter hypoplasia and gliosis. Neurofilament staining (f, inset) indicates an axonal spheroid, which were only seen in the ZIKV-infected fetus. Scale bars, $1 \mathrm{~mm}$ (a,b), $500 \mu \mathrm{m}(\mathbf{c}, \mathbf{d}), 100 \mu \mathrm{m}(\mathbf{e}, \mathbf{f})$ and $50 \mu \mathrm{m}$ (f, inset). (g,h) Representative image from the entire brain section showing GFAP immunostaining in the control (g) or ZIKV-infected fetus (h). (i) ZIKV RNA levels in dam and fetal tissues, as measured by a virus-specific qRT-PCR assay. Data are mean \pm s.d. (j) Schematic of the ZIKV FSS13025 genome (top) and viral sequence reads across the ZIKV FSS13025 genome (GenBank accession number KU955593), as determined by RNA-seq analysis in brain tissues from the ZIKV-infected fetus and dam. The data were compared to that of the mock control, which was the total RNA-seq data from the temporal lobe of a female, uninfected pigtail macaque (Macaca nemestrina) that was obtained through the Nonhuman Primate Reference Transcriptome Resource (http://www.nhprtr.org).

impending microcephaly if given sufficient time, but our experimental duration was relatively short (a single pregnancy trimester). Viral neuro-invasion was supported by detection of ZIKV RNA in the fetal and maternal brain, histopathology and fetal serology. The highest copy number of ZIKV RNA was found in placental chorionic villous tissue, which may be the source of the prolonged viremia that has been described in both pregnant humans and rhesus macaques ${ }^{10,11}$. In summary, clinical findings and evidence for viral neuro-invasion is highly consistent with vertical transmission, congenital ZIKV infection and fetal brain injury in our model.
In contrast to our findings, a prior study of ZIKV infection in pregnant rhesus macaques did not observe changes in fetal brain growth; ZIKV RNA was detected in only a few fetal organs (lymph node, bone marrow and optic nerve) in one of three experiments (https:// zika.labkey.com/project/home/begin.view?; last accessed August 1, $2016)^{11}$. Discrepancies may be due to differences in the nonhuman primate species used and the inoculum dose. We chose an inoculum dose based on a study assessing viral load of WNV; during a mosquito bite, inoculated virus ranges from $10^{2}-10^{7}$ infectious viral particles ${ }^{12}$. Mosquitoes typically deposit virus several times as they 'probe' 
before they 'bite'. A prior study also indicated a viral load of up to $10^{8}$ tissue culture infectious dose $50\left(\mathrm{TCID}_{50}\right)$ in the salivary gland of the Aedes aegypti mosquito ${ }^{13}$. Thus, we chose a viral dose at the higher end of the spectrum to mimic the multiple probes and bite of an infected, feeding mosquito.

We describe the first case of fetal brain injury in a nonhuman primate after maternal infection with ZIKV. Arrested fetal brain growth, white matter injury and detection of ZIKV RNA in multiple fetal organs (brain, liver and placenta) are similar to features of the congenital ZIKV syndrome in humans. The distribution of white matter injury in this case is predicted to result in visual-field loss (injury to optic tracts) and neurodevelopmental delay ${ }^{14}$. A similar but more severe pattern of white matter injury, with involvement of the occipital horns of the lateral ventricle, was also seen in a Brazilian child with congenital ZIKV infection (Supplementary Fig. 8). We did not observe many of the severe findings associated with the congenital ZIKV syndrome, but we speculate that this may be due to terminating the study 6 weeks after inoculation because the pregnancy was nearing the due date.

Nonhuman primates are ideal for studying infection in pregnancy because they most closely mimic human gestation in many key respects, including placentation, gray/white matter ratios in the brain and timing of neurodevelopment ${ }^{15}$. The pigtail macaque is known to be susceptible to multiple Flaviviridae family members (such as DENV, Japanese encephalitis virus and hepatitis $\mathrm{C}$ virus), which makes them an excellent model for ZIKV infection ${ }^{16-19}$. In summary, we demonstrate that a clinically inapparent maternal ZIKV infection during pregnancy has the capacity to result in fetal brain injury in a pregnant pigtail macaque. Further studies are needed to investigate the mechanisms of fetal injury. The pigtail macaque model may have significant utility for testing novel vaccines and therapeutics to prevent the congenital ZIKV syndrome.

\section{METHODS}

Methods and any associated references are available in the online version of the paper.

Accession codes. Gene Expression Omnibus: all sequencing data are available under accession code GSE84614.

Note: Any Supplementary Information and Source Data files are available in the online version of the paper.

\section{ACKNOWLEDGMENTS}

We thank the Seattle Genomics Core Laboratory of the Washington National Primate Research Center Systems Biology Division for sequence analysis and the Center for Innate Immunity and Immune Disease immuno-informatics core group for bioinformatics support. We would like to acknowledge J. Hamanishi for technical assistance with preparation of the figures and L. Brandão (neuroradiologist at the Clínica Felippe Mattoso) for providing images and clinical information from a child with congenital ZIKV syndrome.
We thank R. Tesh (University of Texas Medical Branch at Galveston-World Reference Center for Emerging Viruses and Arboviruses) for providing viral seed stocks. We thank A. Powers (US Centers for Disease Control and Prevention) for technical guidance for the qPCR experiments and C. Hughes for administrative assistance. We also thank M.-T. Little for technical editing. This work was supported by funding from the University of Washington Department of Obstetrics and Gynecology (K.M.A.W.) and the US National Institutes of Health (NIH) (grant no. R01AI100989 (L.R. and K.M.A.W.), AI104002 (M.G.), AI083019 (M.G.), R01EB017133 (C.S.), R01NS055064 (C.S.) and R01NS061957 (C.S)). The NIH training grants T32 HD007233 and T32 AI07509 provided support for E.B. and J.V., respectively. This project was also supported by the Office of Research Infrastructure Programs (ORIP) of the NIH through grant no. P51OD010425 to the Washington National Primate Research Center (A.B., R.F.G., L.K., J.O., G.M.G., W.L. and C.E.). The content of this paper is solely the responsibility of the authors and does not necessarily represent the official views of the US National Institutes of Health or other funders. The funders had no role in study design, data collection and analysis, the decision to publish or preparation of the manuscript.

\section{AUTHOR CONTRIBUTIONS}

K.M.A.W., J.E.S.-B., R.P.K., C.S., M.G. and L.R. designed the study; K.M.A.W., J.E.S.-B., R.P.K., C.S., E.B., J.V., A.B., M.K.D., J.T., S.M., B.A., J.T.-G., M.A.D., E.C.D., M.R.F., J.C.G., T.R., M.S.D., L.K., J.O., G.M.G., W.L., C.E. and L.R. performed the experiments; K.M.A.W., J.E.S.-B., R.P.K., C.S., E.B., J.V., A.B., M.K.D., J.T., R.R.G., S.M., B.A., J.T.-G., M.A.D., E.C.D., M.R.F., J.C.G., T.R., G.A.G., S.E.J., R.F.G., L.K., D.W.W.S., R.F.H., W.B.D., M.G. and L.R. analyzed the data; K.M.A.W., J.E.S.-B., R.P.K., C.S., E.B., J.V., M.K.D., J.T., R.R.G, T.R., M.S.D., D.W.W.S., R.F.H., M.G. and L.R. drafted the manuscript; and all authors reviewed the final draft of the manuscript.

\section{COMPETING FINANCIAL INTERESTS}

The authors declare competing financial interests: details are available in the online version of the paper.

Reprints and permissions information is available online at http://www.nature.com/ reprints/index.html.

1. Petersen, L.R., Jamieson, D.J., Powers, A.M. \& Honein, M.A. N. Engl. J. Med. 374, 1552-1563 (2016).

2. Miner, J.J. et al. Cell 165, 1081-1091 (2016).

3. Cugola, F.R. et al. Nature 534, 267-271 (2016).

4. Wu, K.Y. et al. Cell Res. 26, 645-654 (2016).

5. Rasmussen, S.A., Jamieson, D.J., Honein, M.A. \& Petersen, L.R. N. Engl. J. Med. 374, 1981-1987 (2016).

6. Conrad, S., Ha, J., Lohr, C. \& Sackett, G. Am. J. Primatol. 36, 15-35 (1995).

7. Haddad, J. et al. Neuropediatrics 21, 215-217 (1990).

8. Leonard, J.R. et al. AJR Am. J. Roentgenol. 174, 1651-1655 (2000).

9. Brasil, P. et al. N. Engl. J. Med. http://dx.doi.org/10.1056/NEJMoa1602412 (2016).

10. Driggers, R.W. et al. N. Engl. J. Med. 374, 2142-2151 (2016).

11. Dudley, D.M. et al. Nat. Commun. 7, 12204 (2016).

12. Styer, L.M. et al. PLoS Pathog. 3, 1262-1270 (2007).

13. Li, M.I., Wong, P.S., Ng, L.C. \& Tan, C.H. PLoS Negl. Trop. Dis. 6, e1792 (2012).

14. Lennartsson, F., Nilsson, M., Flodmark, O. \& Jacobson, L. Invest. Ophthalmol. Vis. Sci. 55, 8278-8288 (2014).

15. Adams Waldorf, K.M., Rubens, C.E. \& Gravett, M.G. BJOG 118, 136-144 (2011).

16. Bruce, A.G. et al. J. Virol. 87, 13676-13693 (2013).

17. Nakgoi, K. et al. Am. J. Primatol. 76, 97-102 (2014).

18. Putaporntip, C. et al. Mol. Ecol. 19, 3466-3476 (2010).

19. Sourisseau, M. et al. Gastroenterology 145, 966-969.e7 (2013). 


\section{ONLINE METHODS}

Primate experiments. The nonhuman primate experiments were carried out in strict accordance with the recommendations in the Guide for the Care and Use of Laboratory Animals ${ }^{20}$ of the National Research Council and the Weatherall report ${ }^{21}$. The Institutional Animal Care Use Committee of the University of Washington approved the study (permit number: 4165-02). We inoculated ZIKV (strain FSS13025, Cambodia 2010) subcutaneously in five separate locations on the forearms, each with $10^{7}$ plaque-forming units (p.f.u.) into a healthy pregnant, 9 -year-old pigtail macaque (M. nemestrina) at $119 \mathrm{~d}$ gestation ( 28 weeks of human pregnancy) to test directly whether ZIKV causes fetal brain injury. At the time of cesarean section and fetal necropsy, the fetus was at $162 \mathrm{~d}$ gestation (term pregnancy $=172 \mathrm{~d}$ ).

Definition of fever in the pigtail macaque. The definition of a fever in a pigtail macaque is not standardized. A range of rectal temperatures (from 36.6 to $40.1^{\circ} \mathrm{C}$; average $\left.38.8{ }^{\circ} \mathrm{C}\right)^{22}$ has been reported in sedated rhesus and cynomolgus macaques (M. mulatta and M. fascicularis), which are close relatives to the pigtail macaque. Individual variation is thought to relate to excitement before sedation and lowering of body temperature during sedation. The highest maternal rectal temperature recorded in the study was $38.2^{\circ} \mathrm{C}\left(100.8^{\circ} \mathrm{F}\right)$, which was not thought to represent a fever.

Ultrasound. Fetal dates were determined initially by measurement of the crown rump at $45 \mathrm{~d}$; ultrasound measurements of the biparietal diameter (BPD) at $101 \mathrm{~d}$ gestation supported the dating. Thereafter, ultrasound was performed every week from inoculation until delivery. A GE Vivid-i (GE Medical, Waukesha, WI, USA) machine was used for the first ultrasound, and all subsequent measurements were performed on the Philips HD11 machine (Philips Healthcare, Bothell, WA, USA). A curvilinear C5-2 MHz transducer was used to image the fetus. At least three measurements of the head circumference, abdominal circumference, BPD and femur length were acquired, and the average was calculated. In addition, at least three ventricular measurements in the dependent ventricle, transverse cerebellar diameter and placental thickness were acquired, and an average measurement was calculated. At least two cine clips were acquired through the head in an axial and coronal orientation, and through the chest and abdomen in an axial orientation. Doppler ultrasound of the middle cerebral artery was performed to evaluate the flow through the brain. Amniotic fluid index was calculated by measuring the fluid pockets in the four quadrants. Fetal biometry was compared to known standards for the pigtail macaque $^{6}$. Ultrasound was also performed on a control fetus at $163 \mathrm{~d}$ gestation. A board-certified radiologist (M.K.D.) with 13 years of experience in obstetric ultrasound reviewed the imaging.

Magnetic resonance imaging. MR imaging was performed using a Philips Achieva 3T scanner and a multi-element human cardiac radiofrequency (RF) coil. Anesthesia was induced using 5-20 mg per kg body weight ketamine and maintained using no higher than a $3 \%$ sevoflurane-oxygen mixture during the imaging. A three-plane localizer using a balanced fast-field echo (FFE) imaging sequence was used to determine fetal head position. A two-dimensional (2D) single-shot, half-Fourier turbo spin echo multislice sequence was used to acquire T2-weighted images with the following parameters: TR/TE $=2,200 / 160 \mathrm{~ms}$, SENSE acceleration factor $=2$, TSE factor $=100$, along with fat suppression and fold-over artifact suppression. Multiple contiguous $2 \mathrm{D}$ image stacks, with in-plane resolutions of $0.5 \times 0.5 \mathrm{~mm}$ and thicknesses of $2 \mathrm{~mm}$, were acquired along the fetal brain axial, sagittal and coronal axes to facilitate the reconstruction of a $3 \mathrm{D}$ volume with isotropic spatial resolution. Each 2 -mm-thick plane orientation was repeated six times to provide high signal-to-noise ratio in the final combined 3D reconstruction. Diffusion MR imaging data were also acquired but not analyzed in this case report. A pediatric neuroradiologist (D.W.W.S.) and a pediatric geneticist with expertise in human developmental brain disorders (W.B.D.) qualitatively reviewed the MRI images.

To form a single high-resolution 3D T2W image volume for quantitative analysis of tissue brain volume and surface anatomy, all multislice T2-weighted image stacks were imported into the SLIMMER slice motion correction tool ${ }^{23}$ implemented in the rview software (http://rview.colin-studholme.net, version 9.077.155BQT running under Ubuntu release 14) for initial volume-based alignment to correct for gross drift of the fetal head between slice stacks and to define a standardized orientation of the fetal head anatomy. Between-slice motion occurring due to maternal breathing and fetal head drift was then estimated using slice intersection motion correction ${ }^{24}$. Differences in signal intensity arising from drift of the fetal head with respect to the imaging coils during scanning and from intensity variations due to spin history arising from motion between excitation and readout of each individual slice were corrected using slice-intersection-bias correction. Then, all slices were combined using an iterative deconvolution-based super-resolution 3D-scattered data reconstruction technique, using robust Huber-norm outlier rejection of any residual within-slice motion $\operatorname{artifacts}^{25}$. The final de-convolved isotropic spatial sampling resolution of this image was selected to match the in-plane resolution of each slice $(0.5 \mathrm{~mm})$.

Each reconstructed $3 \mathrm{D}$ brain image volume was first masked to exclude skull and maternal tissues. As no age-specific atlas is available for this species, the MR imaging scan was roughly segmented into gray matter, white matter and cerebrospinal fluid (CSF) using an initial $K$-means clustering initiated from a manually selected set of voxels in each tissue class. The output of this was then extensively manually edited using the review segmentation tool (http://rview. colin-studholme.net, version 9.077.155BQT running under Ubuntu release 14) to divide the tissues further into cortical and deep gray matter, white matter, cerebellum, sulcal cerebrospinal fluid and ventricular cerebrospinal fluid. In addition, regions of age-abnormal fluid representing abnormal posterior ventricular CSF regions and any surrounding unconnected regions of high T2W signal were separately labeled as abnormal fluid volume. From these we calculated the total supratentorial cortical gray matter, white matter and deep gray matter, the total cerebellum volume and the abnormal fluid volume in cubic centimeters. The boundary between the gray and white matter voxels was converted to a topologically correct triangular surface mesh with an initial voxel-label topology correction using an implementation of Bazin et al. ${ }^{26}$ and then tessellated using an implementation of the marching cubes algorithm. This inclusive whole-brain surface includes the regions of the hemispheric connections and the truncation surface at the brain stem. The area of all triangles in the surface was then summed to create a single surface area estimate for the brain at each time point in square centimeters.

MR spectroscopy (MRS). Single-voxel MRS was acquired using a point-resolved spectroscopy pulse sequence (voxel size: $15 \times 12.5 \times 15 \mathrm{~mm}$ ) centered on a frontal region and a posterior region. The structural image was reconstructed in real time to guide MRS voxel placement. Acquisition parameters included: repetition time $\mathrm{TR}=2,000 \mathrm{~ms}$; echo time $\mathrm{TE}=32 \mathrm{~ms} ; 2,048$ complex free-induction decay points, and 2,000-Hz spectral width. Absolute concentrations of metabolites: $\mathrm{N}$-acetyl aspartate (NAA), creatine (Cr), choline (Cho), myoinositol (Ins), glutamate $(\mathrm{Glu})$ and glutamate + glutamine (Glx) were calculated using LCModel ${ }^{27}$. Absolute concentrations were obtained by scaling the in vivo spectrum to the unsuppressed water peak and are reported in units that approximate millimolar concentrations. Partial volume corrections were made by calculating the fraction of cerebrospinal fluid for each voxel.

Cultures. Bacterial cultures were performed to exclude microbial infection. Amniotic fluid, and maternal and fetal blood, were collected at necropsy and plated on tryptic soy agar (TSA) plates and incubated up to $48 \mathrm{~h}$ at $37^{\circ} \mathrm{C}$ in $5 \% \mathrm{CO}_{2}$. Maternal and fetal meninges and membranes were swabbed and streaked on TSA plates and incubated up to $48 \mathrm{~h}$ at $37{ }^{\circ} \mathrm{C}$ in $5 \% \mathrm{CO}_{2}$. No bacteria were cultured from any these samples.

Serology for WNV, CHIKV, DENV and ZIKV. We used virus-specific ELISA assays for ZIKV-, WNV-, CHIKV- or DENV-specific IgG to test ultravioletinactivated plasma according to the manufacturer's (Express Biosystems, Frederick, MD) instructions. Diluted plasma samples were added to the wells of the ELISA plate, which was coated with viral antigens, and incubated at $37^{\circ} \mathrm{C}$ for $45 \mathrm{~min}$. Wells were washed five times, and peroxidase conjugate was added to each well and incubated for $45 \mathrm{~min}$ at $37^{\circ} \mathrm{C}$. Following incubation, the plate was washed five times, followed by the addition of $2,2^{\prime}$-azino-bis(3-ethylbenzothiazoline6-sulfonic acid)-peroxidase substrate to each well. The plate was incubated at room temperature for $30 \mathrm{~min}$, and the absorbance of the colorimetric reaction in each well was read within $15 \mathrm{~min}$ on a plate reader at $405 \mathrm{~nm}$. 
Immunohistochemistry. The Seattle Children's Hospital Pathology Department performed the immunohistochemistry for GFAP, cleaved caspase-3, CD3, CD20, CD163 and neurofilament on formaldehyde-fixed paraffin-embedded sections of cerebral cortex from the Zika-exposed fetus and an age-matched control. Immunostaining was performed with an automated immunostainer (Ventana, Tucson, AZ) using the reagents and dilutions shown in Supplementary Table 5. Appropriate peroxidase-conjugated secondary antibodies and diaminobenzidinebased reaction product were used to visualize sites of immunoreactivity. Results were compared to two controls that were close in gestational age (142 and $155 \mathrm{~d}$ gestation).

Cell lines and virus. Vero cells obtained from ATCC were cultured in Dulbecco's modified Eagle's medium (DMEM; Cellgro) supplemented with 10\% FBS (FBS; Hyclone, Logan, UT), $2 \mathrm{mM}$ L-glutamine, $1 \mathrm{mM}$ sodium pyruvate, $100 \mathrm{U} / \mathrm{mL}$ penicillin, $100 \mu \mathrm{g} / \mathrm{mL}$ streptomycin and $1 \times$ non-essential amino acids (Sigma). Zika virus strain FSS13025 was isolated in Cambodia in 2010 and provided by the World Reference Center of Emerging Viruses and Arboviruses (WRCEV, Galveston, Texas, USA). Working stocks were obtained by plaque-purifying virus and amplifying once in Vero cells. Virus was adsorbed to cells in DMEM supplemented to contain $1 \% \mathrm{FBS}$ at $37^{\circ} \mathrm{C}$ for $2 \mathrm{~h}$. The inoculum was removed, and the virus was propagated in medium supplemented to contain $5 \% \mathrm{FBS}, 2 \mathrm{mM}$ L-glutamine, $1 \mathrm{mM}$ sodium pyruvate, $100 \mathrm{U} / \mathrm{mL}$ penicillin, $100 \mu \mathrm{g} / \mathrm{mL}$ streptomycin, $20 \mathrm{mM}$ HEPES and $1 \times$ non-essential amino acids (Sigma). Supernatants were collected and spun at 2,000 r.p.m. at $4{ }^{\circ} \mathrm{C}$ for $10 \mathrm{~min}$ and frozen in aliquots at $-80^{\circ} \mathrm{C}$. All of the cell lines used lacked mycoplasma.

Measurement of viral RNA load. Viral RNA load was assessed in tissues from the dam, fetus and placenta using a ZIKV-specific RT-qPCR assay. Fetal and maternal organs were placed in RNA-later (Qiagen, Valencia, CA) immediately after harvest. For analysis of Zika virus RNA in tissue, organs were weighed and homogenized in buffer RLT (Qiagen, Valencia, CA) using a bead-beater apparatus (Precellys, Bertin Corp., Rockville, MD). RNA was extracted from tissues using the RNeasy kit (Qiagen, Valencia, CA). Following RNA extraction, cDNA was synthesized using the iScript select cDNA synthesis kit (Bio-Rad, Hercules, CA) according to manufacturer's protocols for gene-specific primers. Viral RNA was quantified using the Taqman Universal Master Mix (Applied Biosystems, Waltham, MA) and an Applied Biosystems 7300 RT-PCR machine using primers previously described ${ }^{28}$. Primer sequences listed in Supplementary Table 6 correspond to residues in the FSS13025 genome (GenBank accession number KU955593). A standard curve was generated by serially diluting a plasmid containing residues 699-2,382 of the Zika virus genome. The standard curve was used to extrapolate the number of copies of Zika virus RNA per mg of tissue. To adhere to stringent guidelines, $C_{\mathrm{t}}$ (cycle threshold) values $>38$ were deemed as not reliably detected and were not reported.

Plaque assay. Monolayers of Vero cells were plated in 6-well plates. Tissue homogenates, CSF or viral stocks were serially diluted in DMEM supplemented to contain $1 \%$ FBS. These dilutions were adsorbed to monolayers of Vero cells at $37^{\circ} \mathrm{C}$ for $2 \mathrm{~h}$. The inoculum was replaced with a $1 \%$ agarose overlay, as previously described for West Nile Virus ${ }^{29}$. Five days following inoculation, a second $1 \%$ agarose overlay containing $2 \%$ neutral red (Sigma) was added to the cells. Plaques were counted $4 \mathrm{~h}$ after the second overlay.

Library construction and transcriptome sequencing. Ribosomal RNA (rRNA) was depleted from each RNA sample using the Ribo-Zero rRNA Removal Kit (Epicentre, Madison, WI) following the manufacturer's instructions. Libraries were prepared from $150 \mathrm{ng}$ of rRNA-depleted RNA following the KAPA Stranded RNA-seq with RiboErase workflow for Total RNA-seq libraries (KAPA Biosystems, Wilmington, MA). Library quality was evaluated using the Qubit 3.0 Fluorometer and the Agilent 2100 Bioanalyzer instrument. Constructed libraries were sequenced on a NextSeq 500 Illumina platform, producing $2 \times 75$ nucleotide stranded paired-end reads $(52 \mathrm{~Gb})$.

Data processing and viral read mapping. Quality control of the primary sequencing data was performed using FastQC (version 0.11.3) and included adaptor trimming using cutadapt (version 1.8.3). FastQC showed good quality statistics across all samples. Ribosomal RNA reads were removed computationally using Bowtie2 with an index composed of human, mouse and rat rRNA sequences. Sequence reads were trimmed to $50 \mathrm{bp}$ and then aligned to the reference FSS13025 genome (Genbank number: KU955593) using Bowtie2 with default settings. Viral read alignment was visualized using the Integrative Genomics Viewer (IGV) tool ${ }^{30,31}$. Primary Total RNA-seq data from the temporal lobe of a female, uninfected pigtail macaque (M. nemestrina) was obtained through the Nonhuman Primate Reference Transcriptome Resource (http:// www.nhprtr.org) to serve as a control ${ }^{32}$. All primary sequence data are available at Sequence Read Archive (SRA) under the Gene Expression Omnibus (GEO) accession GSE84614. Raw sequence data was uploaded to the GEO and SRA databases (GSE84614).

Flow cytometry. Flow cytometry was performed on maternal and fetal brain tissues. To generate single-cell suspensions of brain tissue, each tissue was filtered through a $280-\mu \mathrm{m}$ metal sieve, pelleted at $500 \mathrm{~g}$ for $5 \mathrm{~min}$, washed once in sterile PBS, resuspended in FACS buffer (1 mM EDTA, 25 mM HEPES and 0.1\% BSA $(\mathrm{wt} / \mathrm{vol})$ in PBS) and placed on ice until staining. Single-cell suspensions were pelleted at $500 \mathrm{~g}$ for $5 \mathrm{~min}$ and diluted to $\sim 10^{7}$ cells $/ \mathrm{mL}$ in FACS buffer. Cell suspensions $(100 \mu \mathrm{L})$ were treated with Fc block (1:200; BD Biosciences) for 15 min at $4{ }^{\circ} \mathrm{C}$. After Fc block treatment, cells were stained with anti-CD56 (1:10; clone B159) for $1 \mathrm{~h}$ at $4{ }^{\circ} \mathrm{C}$ (all antibodies are from BD Biosciences unless noted otherwise). Cells were washed twice in FACS buffer to remove excess antibody, fixed for $15 \mathrm{~min}$ at $4{ }^{\circ} \mathrm{C}$ with $2.0 \%$ paraformaldehyde, then permeabilized in $0.1 \%$ (vol $/ \mathrm{vol})$ Triton X-100 for $15 \mathrm{~min}$ at room temperature. Following permeabilization, cells were washed once in FACS buffer to remove Triton X-100 and stained with anti-GFAP (1:10; clone REA335; Miltenyi Biotec) for $1 \mathrm{~h}$ at $4{ }^{\circ} \mathrm{C}$. After intracellular staining, cells were washed twice in FACS buffer and analyzed using an LSRII flow cytometer (BD Biosciences). Cell surface and intracellular markers were analyzed using FlowJo software version 10.1 (FlowJo). Unstained and single-color compensation beads (BD Biosciences) were included for compensation.

Approval for de-identified human subjects. The Seattle Children's Hospital Institutional Review Board for Protection of Human Subjects approved the use of de-identified MRI images.

20. National Research Council. Guide for the Care and Use of Laboratory Animals 8th edn. (The National Academies Press, 2011).

21. Weatherall, D. The use of nonhuman primates in research. (Academy of Medical Sciences, Medical Research Council, The Royal Society and Wellcome Trust, 2006).

22. Woods, S.E., Marini, R.P. \& Patterson, M.M. J. Am. Assoc. Lab. Anim. Sci. 52 295-300 (2013).

23. Kim, K. et al. IEEE Trans. Med. Imaging 29, 146-158 (2010).

24. Kim, K. et al. IEEE Trans. Med. Imaging 30, 1704-1712 (2011).

25. Fogtmann, M. et al. IEEE Trans. Med. Imaging 33, 272-289 (2014).

26. Bazin, P.L. \& Pham, D.L. Comput. Methods Programs Biomed. 88, 182-190 (2007).

27. Provencher, S.W. NMR Biomed. 14, 260-264 (2001).

28. Lanciotti, R.S. et al. Emerg. Infect. Dis. 14, 1232-1239 (2008).

29. Keller, B.C. et al. J. Virol. 80, 9424-9434 (2006).

30. Robinson, J.T. et al. Nat. Biotechnol. 29, 24-26 (2011).

31. Thorvaldsdóttir, H., Robinson, J.T. \& Mesirov, J.P. Brief. Bioinform. 14, 178-192 (2013).

32. Pipes, L. et al. Nucleic Acids Res. 41, D906-D914 (2013). 